\title{
New Exact Periodic Wave Solutions of the Nonlinear Klein-gordon Equation
}

\author{
Hao Jianzhong, Zhuo Li , Wang Ting , Liu Jinbo, Liu Gang \\ Fundamental Department of Xuzhou Air Force College, Xuzhou, 221000, China \\ E-mail: hjz0516@163.com
}

\begin{abstract}
To the non-linear Klein-Gordon equations, the undetermined assumption method is used to get the exact periodic wave solution in the form of Jacobi elliptic function fraction and with the asymptotic values non-zero. The condition for their existence and boundness are obtained and the impact of the change of travelling periodic wave velocity is revealed upon the periodic wave solution and the size of the period.
\end{abstract}

Keywords-Nonlinear Klein-Gordon equation, Jacobi elliptic function, Periodic wave solutions

\section{INTRODUCTION}

Non-linear Klein-Gordon equation ${ }^{[1]}$

$$
u_{t t}-u_{x x}+\lambda u+\mu u^{3}=0
$$

is an important model in mechanics and particle physics. In $[3,4]$, by expansion to the Jacobi elliptic function, the exact periodic solution is got to the equation in the following form.

$$
u(\xi)=\sum_{i=0}^{n} a_{i} s n^{i}(\xi)+\sum_{j=1}^{n} b_{j} s n^{-j}(\xi)
$$

Here, we try to give the periodic wave solution to the non-linear Klein-Gordon equation (1) with the form as

$$
x(\xi)=\left[\frac{A_{0}+A_{1} c n^{2}(\alpha \xi, k)}{B_{0}+B_{1} c n^{2}(\alpha \xi, k)}\right]^{\frac{1}{2}}
$$

and get to know the existence and bound condition of the solution.

\section{NEW EXACT PERIODIC WAVE SOLUTION TO THE NON-LINEAR KLEIN-GORDON EQUATION}

It is easily known that the travelling wave solution of equation (1) $u(x, t)=u(\xi)=u(x-v t)$ satisfies,

$$
\begin{array}{rlrl} 
& & \left(v^{2}-1\right) u^{\prime \prime}(\xi)+\lambda u+\mu u^{3} & =0 \\
\text { or } & u^{\prime \prime}(\xi)+l u+s u^{3} & =0
\end{array}
$$

in which, $l=\frac{\lambda}{v^{2}-1}, s=\frac{\mu}{v^{2}-1}$

Let $u(\xi)=\sqrt{p(\xi)}$, then equation (3) can be changed as

$$
\frac{1}{2} p(\xi) p^{\prime \prime}(\xi)-\frac{1}{4}\left(p^{\prime}(\xi)\right)^{2}+l p^{2}(\xi)+s p^{3}(\xi)=0 \text {. }
$$

To get the solution to equation (1), we just need the solution to equation (3), which is the positive solution to equation (4). Let equation (4) have the solution with the form as

$$
p(\xi)=\frac{A_{0}+A_{1} c n^{2}(\alpha \xi, k)}{B_{0}+B_{1} c n^{2}(\alpha \xi, k)} \quad(\mathrm{k} \text { is module })
$$

From (5), we can easily get the expression of $p^{\prime}(\xi), p^{\prime \prime}(\xi)$, and then put the expressions and (5) into (4). After simplification, and by comparing the coefficients of the powers of $\operatorname{cn}(\alpha \xi, k)$ and setting them as zero, we get the following equations,

$$
\left\{\begin{array}{c}
A_{0} B_{0}\left(k^{2}-1\right)\left(A_{0} B_{1}-A_{1} B_{0}\right) \alpha^{2}+A_{0}^{2}\left(A_{0} B_{0} s+B_{0}^{2} l\right)=0 \\
2 A_{0}\left(2 B_{0} k^{2}-B_{0}+B_{1} k^{2}-B_{1}\right)\left(A_{1} B_{0}-A_{0} B_{1}\right) \alpha^{2} \\
\quad+2 A_{0} B_{0}\left(A_{0} B_{1}+A_{1} B_{0}\right) l+A_{0}^{2}\left(A_{0} B_{1}+3 A_{1} B_{0}\right) s=0 \\
\left(A_{0} B_{1}-A_{1} B_{0}\right)\left(\left(2 A_{0} B_{1}-3 A_{1} B_{1}-2 A_{1} B_{0}+3 A_{0} B_{0}\right) k^{2}-A_{0} B_{1}\right. \\
\left.\quad+3 A_{1} B_{1}+A_{1} B_{0}\right) \alpha^{2}+\left(A_{0}^{2} B_{1}^{2}+4 A_{0} A_{1} B_{0} B_{1}+A_{1}^{2} B_{0}^{2}\right) l \\
+3 A_{0} A_{1}\left(A_{0} B_{1}+A_{1} B_{0}\right) s=0 \\
2 A_{1}\left(B_{0} k^{2}+2 B_{1} k^{2}-B_{1}\right)\left(A_{0} B_{1}-A_{1} B_{0}\right) \alpha^{2} \\
\quad+2 A_{1} B_{1}\left(A_{1} B_{0}+A_{0} B_{1}\right) l+A_{1}^{2}\left(A_{1} B_{0}+3 A_{0} B_{1}\right) s=0 \\
A_{1} B_{1} k^{2}\left(A_{0} B_{1}-A_{1} B_{0}\right) \alpha^{2}-A_{1}^{2}\left(A_{1} B_{1} s+B_{1}^{2} l\right)=0
\end{array}\right.
$$

To solve the equations with the Maple, we get

$$
\begin{aligned}
A_{0} & =0, B_{0}=\frac{2 y\left(1-2 k^{2}\right)(l y+s)}{(4 l y+3 s) k^{2}} A_{1}, B_{1}=y A_{1}, \\
\alpha^{2} & =\frac{4 l y+3 s}{2 y\left(2 k^{2}-1\right)} .
\end{aligned}
$$

Here y satisfies the equation

$$
4 l^{2} y^{4}+6 l s y^{3}-s^{2}\left(k^{4}-k^{2}-2\right) y^{2}=0 .
$$

Then we get the root of equation (8) 


$$
y_{1}=-\frac{s\left(k^{2}+1\right)}{2 l}, y_{2}=\frac{s\left(k^{2}-2\right)}{2 l}, y_{3,4}=0 .
$$

Put $y_{i}(i=1,2,3,4)$ respectively into (7), we get the expressions of $A_{i}, B_{i}(i=0,1)$ and $\alpha$, and then put them into (5), the solution to equation (4) is obtained. And put it into $u(\xi)=\sqrt{p(\xi)}$, we get the solution of equation (3), and further we get the solution of equation (1). When $y=y_{1}$, there exists

$$
\begin{aligned}
& u_{1}(\xi)= \\
& {\left[-\frac{2 k^{2} \lambda}{\mu\left(1+k^{2}\right)} \cdot \frac{c n^{2}\left(\sqrt{\frac{\lambda}{\left(v^{2}-1\right)\left(1+k^{2}\right)}} \xi, k\right)}{1-k^{2}+k^{2} c n^{2}\left(\sqrt{\frac{\lambda}{\left(v^{2}-1\right)\left(1+k^{2}\right)}} \xi, k\right)}\right]^{\frac{1}{2}}}
\end{aligned}
$$

Note: In the computation, just omit the meaningless solutions and the solutions determined as boundless according to the nature of Jacobi ellipse function or with $p(\xi)$ less than zero. We do it the way in the following and will not repeat this.

2)

$$
\begin{aligned}
& A_{0}=-A_{1}, B_{0}=\frac{y\left(k^{2}-1\right)\left(2 k^{2} l y-2 l y+k^{2} s-2 s\right)}{s\left(1+k^{2}\right) k^{2}}, \\
& B_{1}=y A_{1}, \alpha^{2}=-\frac{s^{2}\left(k^{2}+1\right)}{2 y\left(k^{2}-1\right)^{2}(4 l y+3 s)} .
\end{aligned}
$$

in which y satisfies the equation

$$
\begin{aligned}
& 4 l^{2}\left(k^{2}-1\right)^{2} y^{4} \\
& +6 l s\left(k^{2}-1\right)^{2} y^{3}+\left(2 k^{4}-5 k^{2}+2\right) s^{2} y^{2}=0 .
\end{aligned}
$$

Then we easily get the root of equation (10)

$$
y_{1}=\frac{\left(2-k^{2}\right) s}{2\left(k^{2}-1\right) l}, \quad y_{2}=\frac{\left(1-2 k^{2}\right) s}{2\left(k^{2}-1\right) l}, \quad y_{3,4}=0 \text {. }
$$

As the above mentioned, here when $y=y_{2}$, there exists $u_{2}(\xi)=$

$$
\left[\frac{2 k^{2} \lambda\left(1-k^{2}\right)}{\mu\left(1-2 k^{2}\right)} \cdot \frac{1-c n^{2}\left(\sqrt{\frac{\lambda}{\left(1-2 k^{2}\right)\left(v^{2}-1\right)}} \xi, k\right)}{1-k^{2}+k^{2} c n^{2}\left(\sqrt{\frac{\lambda}{\left(1-2 k^{2}\right)\left(v^{2}-1\right)}} \xi, k\right)}\right]^{\frac{1}{2}}
$$

3)

$$
A_{0}=\frac{2 l^{2}-2\left(k^{4}-k^{2}+1\right) y^{2}}{3 k^{2} s y} B_{1},
$$

$$
A_{1}=0, B_{0}=-\frac{2 k^{2} y-y+l}{3 k^{2} y} B_{1}, \alpha^{2}=y .
$$

Here y satisfies the following equation

$$
\begin{aligned}
& \left(k^{2}-2\right)\left(2 k^{2}-1\right)\left(k^{2}+1\right) y^{3} \\
& -3 l\left(k^{4}-k^{2}+1\right) y^{2}+l^{3}=0 .
\end{aligned}
$$

We can easily get the root of equation (12),

$$
y_{1}=\frac{l}{k^{2}+1}, y_{2}=\frac{l}{1-2 k^{2}}, y_{3}=\frac{l}{k^{2}-2} .
$$

And when $y=y_{3}$, there exists

$u_{3}(\xi)=$

$\left[\frac{2\left(k^{2}-1\right) \lambda}{\mu\left(2-k^{2}\right)} \cdot \frac{1}{1-k^{2}+k^{2} c n^{2}\left(\sqrt{\frac{\lambda}{\left(k^{2}-2\right)\left(v^{2}-1\right)}} \xi, k\right)}\right]^{\frac{1}{2}}$

4)

$$
\begin{aligned}
& A_{0}=0, A_{1}=\frac{2 l k^{2}}{s\left(1-2 k^{2}\right)} B_{0}, \\
& B_{1}=0, \alpha^{2}=\frac{l}{1-2 k^{2}} .
\end{aligned}
$$

With the same method, we can directly get $u_{4}(\xi)=$

$$
\begin{aligned}
& {\left[\frac{2 k^{2} \lambda}{\mu\left(1-2 k^{2}\right)} \cdot c n^{2}\left(\sqrt{\frac{\lambda}{\left(1-2 k^{2}\right)\left(v^{2}-1\right)}} \xi, k\right)\right]^{\frac{1}{2}} .} \\
& A_{0}=-\frac{2 l\left(1-k^{2}\right)}{s\left(2-k^{2}\right)} B_{0}
\end{aligned}
$$

5) $A_{1}=-\frac{2 l k^{2}}{s\left(2-k^{2}\right)} B_{0}$,

$$
B_{1}=0, \alpha^{2}=-\frac{l}{2-k^{2}} \text {. }
$$

The same as the above, we can get $u_{5}(\xi)=$

$$
\left[\frac{2 \lambda\left(1-k^{2}+k^{2} c n^{2}\left(\sqrt{-\frac{\lambda}{\left(2-k^{2}\right)\left(v^{2}-1\right)}} \xi, k\right)\right)}{\mu\left(k^{2}-2\right)}\right]^{\frac{1}{2}}
$$




$$
A_{0}=-\frac{2 l k^{2}}{s\left(1+k^{2}\right)} B_{0}
$$

6) $A_{1}=\frac{2 l k^{2}}{s\left(1+k^{2}\right)} B_{0}$,

$$
B_{1}=0, \alpha^{2}=\frac{l}{1+k^{2}} \text {. }
$$

Also we can get

$$
\begin{aligned}
& u_{6}(\xi)= \\
& {\left[-\frac{2 k^{2} \lambda}{\mu\left(1+k^{2}\right)} \cdot\left(1-c n^{2}\left(\sqrt{\frac{\lambda}{\left(1+k^{2}\right)\left(v^{2}-1\right)}} \xi, k\right)\right)\right]^{\frac{1}{2}}}
\end{aligned}
$$

Based on analysis and discussion, we know: $u_{1}(\xi)$ 、 $u_{6}(\xi)$ need to satisfies the condition $\lambda\left(v^{2}-1\right)>0, \mu\left(v^{2}-1\right)<0$, and $u_{2}(\xi)$ 、 $u_{4}(\xi)$ need to satisfies the condition $\lambda\left(1-2 k^{2}\right)\left(v^{2}-1\right)>0, \mu\left(v^{2}-1\right)>0$ while $u_{3}(\xi), u_{5}(\xi)$ need the condition $\lambda\left(v^{2}-1\right)<0, \mu\left(v^{2}-1\right)>0$.

\section{DISCUSSION ON THE PERIODIC WAVE SOLUTION TO EQUATIONS WITH THE FORM AS (2)}

For convenience to discussion, we take it for example that equation (1) satisfies conditions

$$
\lambda\left(v^{2}-1\right)>0, \mu\left(v^{2}-1\right)<0
$$

which are the conditions that $u_{1}(\xi) 、 u_{6}(\xi)$ satisfy.

When $\lambda>0, \mu<0$, obviously $v^{2}>1$, then equation
(1) has the large-velocity periodic wave solution $u_{1}(\xi)$ 、 $u_{6}(\xi)$ while, when $\lambda<0, \mu>0$, obviously $v^{2}<1$, the equation has small-velocity periodic wave solution $u_{1}(\xi)$ 、 $u_{6}(\xi)$. Analysis shows that the waveform and cycle of the periodic wave solution are influenced by its velocity $v$ and that the bigger the velocity of the periodic wave solution, the bigger the period becomes with the change in the waveform.

\section{REFERENCES}

[1] DOOD .R. K .Solitons and Nonlinear Waves Equations[M]. London. Academic Prec Inc Ltd, 1982.

[2] W.G.Zhang; Q.S.Chang; E.G. Fan. Methods of judging shape of solitary wave and solution formulae for some evolution equations with nonlinear terms of high order[J]. J.Math.Anal.Appl.,2003, 287:1-18.

[3] P.Li,Z.L.Pan. A new development on Jacobian elliptic function expansion method[J], Phys.Lett.A, 2004,332:39-48.

[4] H.Q.Zhang. Extended Jacobi elliptic function expansion method and its applications $[\mathrm{J}]$. Communication in Nonlinear Science and Numerical Simulation, 2005.

[5] XU Gui-Qiong; LI Zhi-Bin;Applications of Jacobi Elliptic Function Expansion Method for Nonlinear Differential-Difference Equations. COMMUNICATIONS IN THEORETICAL PHYSICS,2005,43(3),

[6] D. Baldwin. Symbolic computation of hyperbolic tangent solutions for nonlinear differential-difference equations . Computer Physics Communications. 2004(3),203-217

[7] Wen XY; Lu DZ. Extended Jacobi elliptic function expansion method and its application to nonlinear evolution equation. CHAOS SOLITONS \& FRACTALS, 2009, 41 (3): 1454

[8] ZHAO Qiang;LIU Shi-Kuo; FU Zun-Tao.Exact Periodic-Wave Solutions for $(2+1)$-Dimensional Boussinesq Equation and (3+1)-Dimensional KP Equation. COMMUNICATIONS IN THEORETICAL PHYSICS, 2004,42 (8)

[9] Li Shaowei; Zhang Weiguo. New Soliton Solutions and Periodic Wave Solutions with Cosine Function Form for the Generalized Pochhammer-Chree Equation. MATHEMATICA APPLICATA. 2009, 22(3), 